Instabilities in supersonic compression ramp flow

Fletcher, A. J. P. and Ruban, A. I. and Walker, J. D. A. 2004

MIMS EPrint: 2006.281

Manchester Institute for Mathematical Sciences

School of Mathematics

The University of Manchester

\footnotetext{
Reports available from: http://eprints.maths.manchester.ac.uk/

And by contacting: The MIMS Secretary

School of Mathematics

The University of Manchester

Manchester, M13 9PL, UK
} 


\title{
Instabilities in supersonic compression ramp flow
}

\author{
By A. J. P. FLETCHER ${ }^{1}$, A. I. RUBA N ${ }^{2}$ \\ AND J. D. A. WA L KER ${ }^{1} \dagger$ \\ ${ }^{1}$ Department of Mechanical Engineering and Mechanics, Lehigh University, 19 Memorial Drive West, \\ Bethlehem, PA 18015, USA \\ ${ }^{2}$ Department of Mathematics, University of Manchester, Oxford Road, \\ Manchester M13 9PL, UK
}

(Received 29 January 2003 and in revised form 15 June 2004)

Separation of a supersonic boundary layer near a compression ramp is considered in the limit of large Reynolds numbers and for Mach numbers $O(1)$. When the ramp angle is small, the motion may be described by the well-known triple-deck theory describing viscous-inviscid interactions. For small values of the scaled ramp angle, steady stable solutions can be obtained. However, it is shown that when a recirculation zone is present and the ramp angle is sufficiently large, the flow in the recirculation zone is susceptible to convective instabilities when perturbations are introduced there. At still larger values of the scaled ramp angle, an absolute instability is shown to occur that leads to a violent local breakdown of the boundary layer. The calculated results are shown to be consistent with a theoretical criterion that is the necessary and sufficient condition for the onset of instability.

\section{Introduction}

The classical problem of supersonic boundary-layer flow past a compression ramp has been studied by various authors for more than four decades. In most situations where a solid surface turns toward the flow, a compressive disturbance is induced that gives rise to a viscous-inviscid interaction between the boundary layer and the supersonic external flow. A similar interaction occurs when a shock wave impinges on a boundary layer on a wall. In both situations, a pocket of recirculating flow was observed in several early experimental investigations (Liepmann 1946; Ackeret, Feldmann \& Rott 1947; Chapman, Kuehn \& Larson 1957) in a phenomenon classically described as separation; the recirculation zones were seen to penetrate upstream of the compressive feature (i.e. either an incident shock wave or the corner point of the compression ramp). This behaviour was initially perplexing because the classical boundary-layer equations, with prescribed external pressure gradient, are parabolic and do not permit the propagation of disturbances upstream. The theoretical explanation for the observed phenomena was supplied by the supersonic triple-deck theory of Stewartson \& Williams (1969) and Neiland (1969), who showed that once a compressive disturbance is sufficiently large, nonlinear effects in the boundary layer near the wall will give rise to boundary-layer separation. As a consequence, the induced displacement thickness can act to alter the external flow by inducing a pressure rise ahead of the point of separation, which in turn causes the boundary layer to separate further upstream. This mechanism of upstream influence is now well-understood 
in supersonic boundary layers and extensive reviews exist (e.g. Stewartson 1974; Neiland 1981; Messiter 1983).

Over the years, a number of numerical solutions of the supersonic triple-deck equations have been produced for situations where the ramp angle is small and $O\left(R e^{-1 / 4}\right)$, where $R e$ is the Reynolds number. Rizzetta, Burggraf \& Jenson (1978) and Ruban (1978) showed that once the scaled ramp angle exceeds a certain minimum value, a zone of backflow develops near the corner of the ramp, which spreads in both the upstream and downstream directions as the ramp angle is progressively increased; with increasing ramp angle, it becomes more difficult to produce accurate numerical solutions. The solutions for higher angles clearly show a pressure rise upstream of the corner, followed by a pressure plateau of almost constant pressure near the recirculation zone. Finally a steep pressure rise develops as reattachment occurs and the pressure attains the downstream value. The shear stress on the other hand becomes negative upstream of the corner, plateaus and then increases slightly to a local maximum before falling sharply to an absolute minimum at a location on the ramp. A subsequent and relatively slower rise in the wall shear occurs in the downstream direction. The region where the wall shear falls sharply to a minimum and then increases will be referred to as the wall shear trough.

With increasing ramp angle, the minimum in the wall shear becomes progressively more negative. In addition, the local maximum in wall shear does eventually become positive indicating the evolution of secondary separation zones within a primary recirculation bubble. Smith \& Khorrami (1991) obtained numerical solutions for relatively large scaled angles and argued on the basis of their solutions that a critical angle exists for which the pressure gradient and wall shear develop a singularity. The implication of this hypothesis is that there exists a critical angle above which the triple-deck formulation fails. Recently Korolev, Gajjar \& Ruban (2002) have obtained very accurate solutions of the triple-deck equations for the steady problem using two independent numerical methods and very small mesh sizes; they were able to extend their results to large scaled angles without serious complication and excellent agreement was obtained with a reattachment theory due to Neiland (1970). It was shown that the minimal skin friction in the separation region becomes very large and negative immediately upstream of reattachment as the ramp angle is increased. Furthermore a complex structure develops in the corner region with the primary recirculation zone bifurcating into secondary and then tertiary bubbles. These results do not support the existence of a singularity at finite angle discussed by Smith \& Khorrami (1991) and suggest that the steady triple-deck formulation can be extended to high ramp angles, albeit with increasing difficulty.

Although the steady compression ramp problem is of considerable interest, a question which naturally arises concerns the degree to which these steady solutions are stable to small disturbances. This issue is important because it appears that 'short' separation bubbles, like those observed near compression corners, are observed to apparently break up in experiments, once the ramp angle is sufficiently large, leading to transition to turbulence and a thick turbulent boundary layer on the downstream portion of the ramp. The work of Chapman et al. (1957) established that transitional flow was clearly associated with the bubble and specifically the reattachment zone downstream of the corner. The fine details of the bubble break up are difficult to observe in unsteady supersonic flow but an analogous phenomenon occurs in dynamic stall, for example. As an airfoil is brought to incidence in a uniform stream, a 'short' separation bubble appears on the upper surface at a certain critical angle of attack (see, for example, Degani, Li \& Walker 1996). The bubble is then 
observed to break up with a further increase in angle of attack, leading directly to transition and a turbulent boundary layer on the upper surface of the airfoil (see, for example, Sychev et al. 1998, and the references therein). In both situations, an increase in the controlling parameter leads to local breakdown of the laminar boundary layer.

Cassel, Ruban \& Walker (1995) carried out a series of calculations for progressively higher ramp angles using a time-marching algorithm to solve the unsteady triple-deck equations, starting from an initial configuration where the ramp angle was abruptly changed from zero. The original objective of these computations was to carry the solutions through to a presumed steady state and this was accomplished without complication for relatively low ramp angles. However at a very moderate angle (well below the maximum values considered by Smith \& Khorrami 1991 and Korolev et al. 2002) an apparent absolute instability, $\dagger$ was encountered which precluded carrying out solutions to higher ramp angles. At the time Cassel et al. (1995) went to considerable effort to attempt to confirm that the observed instability was physical, as opposed to numerical in nature. The oscillations that were observed in the wall shear and pressure appeared for all grids considered. The observed instability appeared in the form of a wave packet but because the oscillations were always point-to-point in the grid, it was judged that the computations could not be continued farther in time with good accuracy. All attempts to do so, however, appeared to indicate that once the wave packet had formed, the amplitude of the oscillations did not increase with time. Similar behaviour had been previously observed by Tutty \& Cowley (1986) who calculated the flow over a small bump using a zero displacement interaction law. The onset of the instability seemed to be associated with the first evolution of a point of inflection in some of the velocity profiles in the backflow zone that appeared once the ramp angle exceeded a certain critical value. However the reasoning was speculative and somewhat unsatisfactory, since it did not prove possible to correlate the onset of instability (see also Cassel 1993) with a necessary and sufficient condition for instability described previously by Tutty \& Cowley (1986).

Suppose that $u_{0}(x, y, t)$ denotes the solution for the streamwise velocity in the lower deck of the triple-deck structure, where $x$ and $y$ are the scaled streamwise and normal coordinates and $t$ is the scaled time in the lower deck. Tutty \& Cowley (1986) considered small perturbations about this solution of the form

$$
u(x, y, t)=u_{0}(x, y, t)+\delta \mathrm{e}^{\mathrm{i} k(x-c t)} u_{1}(x, y, t)+\ldots,
$$

with similar expressions for the normal velocity $v(x, y, t)$, the displacement function $A(x, t)$ and the pressure $p(x, t)$. Here $\delta \ll 1$ is the disturbance amplitude, the wave number $k$ is real and the complex wave speed $c=c_{r}+\mathrm{i} c_{i}$. The situation considered was for $k \gg 1$, corresponding to a possible inviscid instability having short wavelengths (compared to the interaction length scales). Tutty \& Cowley (1986) refer to such modes as long-wave Rayleigh modes since they have wavelengths that are large with respect to the boundary-layer thickness. The analysis leads to an eigenrelation of the form

$$
J(x, t, c)=\int_{0}^{\infty} \frac{\mathrm{d} y}{\left\{u_{0}(x, y, t)-c\right\}^{2}}=0
$$

$\dagger$ An absolute instability is one which grows locally and can propagate upstream but is not convected away downstream (see, for example, Huerre \& Monkewitz 1985). 
which serves to determine the complex wave speed $c(x, t)$ for a given streamwise profile $u_{0}$ at any time $t$; note that $u_{0} \rightarrow \infty$ as $y \rightarrow \infty$. The flow is unstable with respect to small disturbances if $c_{i}>0$ at a given streamwise station. As discussed by Tutty \& Cowley (1986), Rayleigh's inflection point theorem and Fjørtoft's theorem are necessary conditions for instability but the condition (1.2) is necessary and sufficient when $c_{i}>0$. Tutty \& Cowley (1986) calculated the eigenvalues $c$ for interactive flows over a bump with one type of interaction law (a zero displacement law) and were able to demonstrate that when a curve of positive $c_{i}$ was evaluated, the evolution of a wave packet was observed. It proved difficult to find a consistent pattern in the results for other interactive laws (S. J. Cowley, private communication). Similar inconclusive results for compression ramp flow were found by Cassel (1993) and Cassel et al. (1995), although the calculated time-dependent results clearly showed the evolution of an instability at sufficiently high ramp angle. At the time, the reason for this apparent inconsistency was not clear.

In the original work of Cassel et al. (1995), it was believed that the most important location to concentrate the grid in the streamwise direction was at the ramp corner and a typical fine mesh computation involved a total of 301 points in the streamwise direction. Recently more capable computers have facilitated similar calculations but at much smaller streamwise grid sizes. In the present study, the computations of Cassel et al. (1995) were repeated but with much more mesh points. It was then discovered that the instabilities evolved earlier when the smaller mesh sizes were used (with up to 3201 points in the streamwise direction). The streamwise meshes used here were found to be small enough to accurately resolve the details of the developing instability. In addition, it was determined that an instability actually develops first in the region of minimal skin friction on the ramp, as opposed to the corner region. As the ramp angle is increased above a first critical angle, a parameter range is encountered where the recirculating flow region in the corner is convectively unstable but the region outside is not. In other words, small perturbations introduced in the recirculating flow grow with time and convect away from the point of disturbance; eventually the growing disturbance is processed by the reattachment zone and is damped out. On the other hand, a disturbance introduced outside the recirculation zone does not grow with time and is simply convected away, provided the initial disturbance amplitude is not too large. In addition, at any streamwise location in the reversed flow region, there appears to be a threshold amplitude, above which the oscillations become unbounded before the disturbance can escape from the recirculation zone.

As the ramp angle is increased to still higher values, a second critical value is reached where instabilities develop in the reversed flow region and slowly but spontaneously grow to relatively large values; at a third and larger critical angle, the disturbance grows to unbounded values leading to local breakdown. Both phenomena are absolute instabilities. This type of evolution seems consistent with other occurrences of absolute instabilities (see, for example, Huerre \& Monkewitz 1985, 1990) where a convective instability first appears as the controlling parameter is increased and then is followed by the occurrence of absolute instability. Consistent patterns in computed positive values of the eigenvalues $c$ began to emerge when the integral in equation (1.2) was evaluated at each streamwise station, but only when the streamwise mesh was sufficiently refined well beyond those employed by Cassel et al. (1995); these results confirm that the evolution of instabilities in the numerical simulations is a physical phenomenon, as opposed to a numerical instability. Finally it is shown that the predicted growth rates associated with $c$ are roughly consistent with those observed in the computations. 


\section{Governing equations}

Consider the flow of an ideal compressible gas with constant specific heat ratio $\gamma$ past a compression ramp. The flow passes at zero incidence over a flat surface, that has a sharp leading edge at $x^{\prime}=0$, and is subsequently deflected through a positive angle $\alpha^{\prime}$ onto a second flat surface at $x^{\prime}=L$. The compression ramp geometry is then of the general form

$$
f_{0}^{\prime}= \begin{cases}\alpha^{\prime}\left(x^{\prime}-L\right), & x^{\prime}>L \\ 0, & x^{\prime} \leqslant L\end{cases}
$$

where $\alpha^{\prime}$ denotes the ramp angle. The supersonic external flow approaching the corner has speed $U_{\infty}$, density $\rho_{\infty}$ and temperature $T_{\infty}$. Let $\mu_{0}$ denote a reference viscosity based on a reference enthalpy of $U_{\infty}^{2}$ and assume that the viscosity is a function of temperature alone given by the power law $\mu^{\prime} / \mu_{0}=\left(h^{\prime} / U_{\infty}^{2}\right)^{n}$, where $n$ is a known constant; here and in the following a prime denotes a dimensional quantity. The Reynolds number and Mach number for the flow are defined by

$$
R e_{0}=\frac{\rho_{\infty} U_{\infty} L}{\mu_{0}}, \quad M_{\infty}=U_{\infty}\left(\frac{\gamma p_{\infty}}{\rho_{\infty}}\right)^{-1 / 2},
$$

respectively. The Reynolds number is assumed large while the Mach number is $O(1)$ for supersonic flow.

Typically, a shock wave is generated at the corner in supersonic flow, and this feature gives rise to an inviscid-viscous interaction with the boundary layer on the wall, which can be calculated using triple-deck theory when the ramp angle is small. The supersonic boundary layer approaching the compression corner has a thickness $O\left(R e_{0}^{-1 / 2}\right)$ and suppose that $\left(u^{\prime}, v^{\prime}\right)$ denote the flow velocities in the streamwise $x^{\prime}$ and normal $y^{\prime}$-directions, respectively. Near the wall and upstream of the interaction region $u^{\prime} \sim \lambda Y$ as $Y=L^{-1} R e_{0}^{1 / 2} y^{\prime} \rightarrow 0$; here $Y$ is the scaled boundary-layer variable and $\lambda$ is a known constant associated with the wall shear just upstream of the corner. If the ramp angle $\alpha^{\prime}$ is gradually increased from zero, a significant interaction with the external flow occurs when $\alpha^{\prime}=O\left(R e_{0}^{-1 / 4}\right)$. The interaction region is a threelayer structure having streamwise extent $O\left(R e_{0}^{-3 / 8}\right)$ centred on the corner with $(a)$ a lower deck having normal thickness $O\left(\operatorname{Re}_{0}^{-5 / 8}\right),(b)$ a middle deck that is the continuation of the upstream boundary layer and has thickness $O\left(R e_{0}^{-1 / 2}\right)$ and $(c)$ an upper deck describing an inviscid region above the boundary layer with thickness $O\left(R e_{0}^{-3 / 8}\right)$.

Defining the $O(1)$ parameter $\beta=\left(M_{\infty}^{2}-1\right)^{1 / 2}$, the appropriate dimensionless scaled variables in the lower deck are

$$
\begin{gathered}
\frac{x^{\prime}}{L}=1+\frac{\beta^{-3 / 4} \lambda^{-5 / 4}}{\mu_{w}^{1 / 4} \rho_{w}^{1 / 2}} R e_{0}^{-3 / 8} x, \quad t^{\prime}=\frac{L}{U_{\infty}} \frac{\beta^{-1 / 2}}{\mu_{w}^{1 / 2} \lambda^{3 / 2}} R e_{0}^{-1 / 4} t, \\
\frac{u^{\prime}}{U_{\infty}}=\frac{\lambda^{1 / 4} \mu_{w}^{1 / 4}}{\beta^{1 / 4} \rho_{w}^{1 / 2}} R e_{0}^{-1 / 8} u+\ldots, \quad \frac{p^{\prime}-p_{\infty}}{\rho_{\infty} U_{\infty}^{2}}=\frac{\lambda^{1 / 2} \mu_{w}}{\beta^{1 / 2}} R e_{0}^{-1 / 4} p+\ldots, \\
\frac{y^{\prime}}{L}=\frac{\beta^{-1 / 4} \mu_{w}^{1 / 4}}{\lambda^{3 / 4} \rho_{w}^{1 / 2}} R e_{0}^{-5 / 8}\left\{y-f_{0}(x)\right\}, \quad \frac{f_{0}^{\prime}}{L}=\frac{\beta^{-1 / 4} \mu_{w}^{1 / 4}}{\lambda^{3 / 4} \rho_{w}^{1 / 2}} R e_{0}^{-5 / 8} f_{0}, \\
\frac{v^{\prime}}{U_{\infty}}=\frac{\beta^{1 / 4} \lambda^{3 / 4}}{\mu_{w}^{-3 / 4} \rho_{w}^{1 / 2}} R e_{0}^{-3 / 8}\left\{v-u \frac{\mathrm{d} f_{0}}{\mathrm{~d} x}\right\}, \quad \alpha^{\prime}=\frac{\mu_{w}^{1 / 2} \beta^{1 / 2}}{\lambda^{-1 / 2}} R e_{0}^{-1 / 4}, \\
\rho^{\prime}=\rho_{\infty} \rho_{w}+\ldots, \quad \mu^{\prime}=\mu_{0} \mu_{w}+\ldots, \quad h^{\prime}=U_{\infty}^{2} g_{w}+\ldots
\end{gathered}
$$


Note that a Prandtl transposition has been incorporated in equations (2.5) and (2.6) and that a subscript $w$ denotes a quantity evaluated at the wall. Substitution of these expansions into the Navier-Stokes equations yields the lower-deck equations in the limit $R e_{0} \rightarrow \infty$ :

$$
\frac{\partial u}{\partial t}+u \frac{\partial u}{\partial x}+v \frac{\partial u}{\partial y}=-\frac{\partial p}{\partial x}+\frac{\partial^{2} u}{\partial u^{2}}, \quad \frac{\partial u}{\partial x}+\frac{\partial v}{\partial y}=0,
$$

with boundary conditions

$$
\begin{gathered}
u=v=0 \quad \text { at } \quad y=0, \quad u \sim y+A(x, t) \text { as } y \rightarrow \infty \text { for all } x, \\
u \sim y \quad \text { as } \quad x \rightarrow \pm \infty \text { for all } y,
\end{gathered}
$$

and the Ackeret interaction law

$$
p=-\frac{\partial A}{\partial x}+\frac{\mathrm{d} f_{0}}{\mathrm{~d} x} .
$$

Here $A(x, t)$ and $p(x, t)$ are the displacement function and pressure, which are to be determined subject to the requirement that both must vanish as $x \rightarrow \pm \infty$. In the subsequent numerical solutions, it is convenient to use a compression ramp of slightly modified geometry (as opposed to equation (2.1)) to avoid possible numerical complications with an abrupt change in slope; let

$$
f_{0}(x)=\frac{1}{2} \alpha\left(x+\sqrt{x^{2}+r^{2}}\right),
$$

which is used throughout with the rounding parameter taken to be $r=0.5$. Other values of $r$ were considered (see also Cassel et al. 1995) but results are similar for small values of $r$.

The error in the triple-deck formulation is $O\left(R e^{-1 / 8}\right)$ but a lower bound to some level of accuracy relative to solutions of the Navier-Stokes equations cannot be established. However, numerical solutions carried out with both formulations (Burggraf et al. 1979) show good agreement with each other, and also experiment down to a Reynolds number of $7 \times 10^{4}$.

It is convenient to recast the interaction problem in terms of the scaled shear stress $\tau=\partial u / \partial y$. Differentiation of the first of equations (2.8) with respect to $y$ eliminates the pressure and leads to the system

$$
\frac{\partial \tau}{\partial t}+u \frac{\partial \tau}{\partial x}+v \frac{\partial \tau}{\partial y}=\frac{\partial^{2} \tau}{\partial y^{2}}, \quad \frac{\partial^{2} \psi}{\partial y^{2}}=\tau,
$$

where the stream function $\psi$ is defined by $u=\partial \psi / \partial y, v=-\partial \psi / \partial x$. The boundary conditions for $\tau$ and $\psi$ follow from equations (2.8)-(2.10) and

$$
\begin{gathered}
\psi=\frac{\partial \psi}{\partial y}=0, \quad \frac{\partial \tau}{\partial y}=\frac{\partial p}{\partial x} \quad \text { at } \quad y=0, \\
\tau \rightarrow 1 \text { as } \quad x \rightarrow \pm \infty \quad \text { or } \quad \text { as } \quad y \rightarrow \infty .
\end{gathered}
$$

The displacement function $A(x, t)$ may be eliminated from the formulation using

$$
A(x, t)=\lim _{y \rightarrow \infty}\{u-y\}=\int_{0}^{\infty}(\tau-1) \mathrm{d} y,
$$


and differentiating the interaction law (2.11) with respect to $x$, it follows that

$$
\left.\frac{\partial \tau}{\partial y}\right|_{y=0}=-\frac{\partial^{2}}{\partial x^{2}}\left(\int_{0}^{\infty}(\tau-1) \mathrm{d} y\right)+\frac{\mathrm{d}^{2} f_{0}}{\mathrm{~d} x^{2}} .
$$

Lastly, it is convenient for numerical solutions to transform the region $-\infty<x<\infty$, $0 \leqslant y<\infty$ into a finite rectangular domain through the affine transformations

$$
\hat{x}=\frac{2}{\pi} \arctan \left(\frac{x-x_{0}}{a}\right), \quad \hat{y}=\frac{2}{\pi} \arctan \left(\frac{y}{b}\right),
$$

where $a$ and $b$ are parameters which control the level of grid packing in physical space and $x_{0}$ is a streamwise location about which mesh clustering is desired. Smaller values of $a$ and $b$ imply more grid points near $x_{0}$ and $y=0$, respectively. It is anticipated (see, for example, Cassel et al. 1995) that the flow in the lower deck in the vicinity of the ramp corner may exhibit a complex structure, and here higher resolution is required as opposed to the linear shear flow that occurs as $x \rightarrow \pm \infty$. Another advantage of the transformations (2.15) is that the need to truncate the solution domain at some large values of $|x|$ or $y$ is eliminated; the domain is defined by $-1 \leqslant \hat{x} \leqslant 1$ and $0 \leqslant \hat{y}<1$. The transformation rules are

$$
\frac{\partial}{\partial x}=\frac{\Gamma(\hat{x})}{a} \frac{\partial}{\partial \hat{x}}, \quad \frac{\partial}{\partial y}=\frac{\Gamma(\hat{y})}{b} \frac{\partial}{\partial \hat{y}},
$$

where $\Gamma(z)=1 / \pi\{1+\cos (\pi z)\}$.

\section{Numerical methods}

The numerical method used to solve the system of equations (2.13) to (2.17) is described by Cassel et al. (1995); it is first-order accurate in time and second-order accurate in the approximations to the spatial derivatives. The solution was started from the flat-plate solution $u=y$ at $t=0$ when the ramp angle is abruptly increased to the desired value. The solutions were then integrated forward in time until either a steady-state was reached or a flow instability was encountered. The condition used to determine whether a steady-state solution had been achieved was that the difference between the wall shear $\tau(\hat{x}, 0)$ at the current time and the previous time divided by the time step $\Delta t$ must be less than $5 \times 10^{-4}$ at each point in the mesh; the quantity tested approximates the time derivative of $\tau(\hat{x}, 0)$ and for those cases which reach a steady state, a monotonic decrease was observed at each point. For those situations where oscillations develop, the time derivative is initially monotonically decreasing at each $\hat{x}$ but, at a time referred to as $t_{d}$, it stared to increase dramatically.

The first-order time differencing employed places a restriction on $\Delta t$ in order that the numerical algorithm remains stable; this can be estimated from a Von Neumann analysis (Ruban 1978; Cassel 1993) as

$$
\Delta t \leqslant \frac{\pi a \Delta \hat{x}}{4\left|u_{\max }\right|}
$$

The maximum velocity occurs at the edge of the inner deck where $u \sim y$. The outer computational boundary was typically taken at $y_{\max }=60$, although calculations were also run for $y_{\max }=100$ and $y_{\max }=150$ to ensure that the location of the outer boundary did not have a significant effect on the computed results. Many of the reported results were run with a mesh concentration parameter $a=5$ and with 801 
mesh points in the streamwise direction and in this case the time-step restriction is $\Delta t \leqslant 1.64 \times 10^{-4}$. For most such computations a time step of $1.25 \times 10^{-4}$ was used; time steps equal to one half and one quarter this value were also used as a check on the accuracy, as well as on the stability of the numerical algorithm.

The calculations carried out by Cassel et al. (1995) at the higher ramp angles considered and at the smallest mesh sizes used were done with 301 and 151 points in the streamwise and normal directions with $a=10$ and $b=5$, respectively, and showed the evolution of a wave packet near the corner point at the end of the shear stress plateau near the local maximum in $\tau(\hat{x}, 0)$. In the present calculations, it was observed that when the number of points in the streamwise direction was increased to 801 , oscillations started at an earlier time farther downstream near the wall shear minimum and along the pressure rise upstream of reattachment. The computations were then rerun with the concentration point shifted from zero to $x_{0}=5$ (that is near the wall shear minimum); in addition the mesh concentration parameters were reduced to $a=b=5$ in order to obtain better resolution in the zone where the wall shear falls sharply from the local maximum to the minimum. Various values of $a$ and $b$ were used as a check on the results and the present values appear to be a reasonable compromise between good resolution near the minimum, while retaining adequate mesh points in the rest of physical space; for example, a value of $a=2.5$ was judged to produce an undesirable skewed mesh distribution. Computational experience showed that the details of the developing instability could only be seen through grid refinement in the streamwise direction. For example a computation with a $(401,151)$ grid and with $x_{0}=5$ for $\alpha=4$ showed barely visible but persistent oscillations in the wall shear trough so that a steady state was never achieved. However, the same case carried out on a $(801,151)$ mesh revealed the formation of an absolute instability, where significant growth and unsteady oscillations $O(1)$ eventually occurred. Unless otherwise noted the results subsequently given here will be based on a $(801,151)$ grid. Note that the computational task is significant for this grid and the time step employed, with a typical computation requiring on the order of a day to complete on a workstation. Note also that in some cases the number of streamwise points was increased to 1601 and 3201 as a check that the computed solutions were indeed grid independent. For the instabilities reported here the normal mesh spacing with 151 points appears to be adequate, since computations were also run with 301 and 601 points but no discernible change in the results was seen.

\section{The absolute instability}

In the instability observed by Cassel et al. (1995), a wave packet was observed to form and persist at an essentially fixed location. Calculated results for this case are shown in figure $1(a)$ for the case $\alpha=4$ with a $(301,151)$ mesh and the same computational parameters employed by Cassel et al. (1995). This pattern evolves in the wall shear stress and pressure around $t=85$ and although there is an oscillation back and forth within an envelope, the behaviour is essentially quasi-steady. In figure 1(a) the mesh is shown in the inset along with a magnified view of the internal structure. Clearly with this streamwise mesh the oscillation is barely resolved. However, the computation can be continued well beyond this stage without additional growth of the wave packet.

When the streamwise mesh was refined and the concentration point moved downstream to $x_{0}=5$ a different and less regular disturbance appears at an earlier time around $t=80$ in the wall shear trough. The pattern shown in figure $1(b)$ is at a 

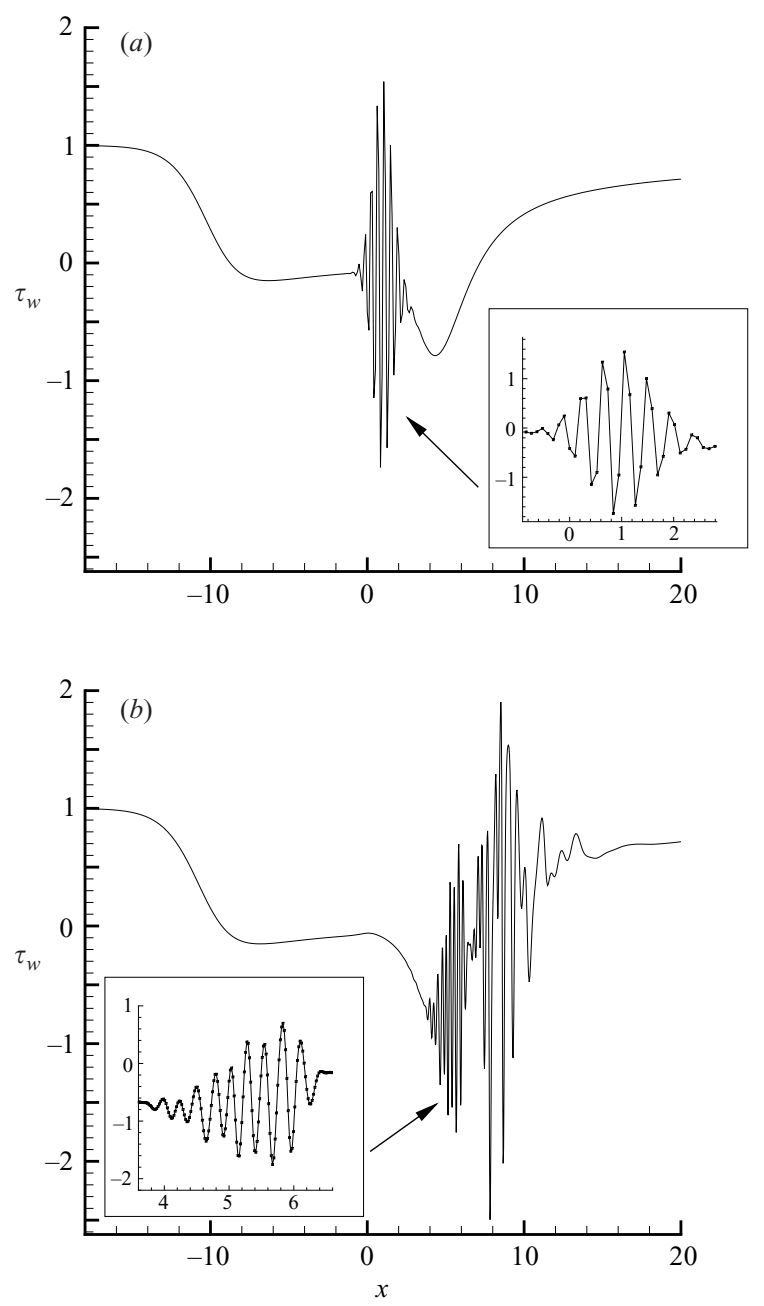

FIGURE 1. Comparison of the evolving disturbance on coarse and refined meshes for $\alpha=4$ : (a) apparent wave packet formed on $(301,151)$ mesh with $a=10, x_{0}=0 ;(b)$ the new disturbance on a $(810,151)$ mesh with $a=5, x_{0}=0$.

particular time when it has grown to a certain level of amplitude. With the passage of time the pattern oscillates back and forth from a location upstream of the minimal wall shear to the reattachment point. The streamwise location of maximum amplitude changes with time but, by and large, the average amplitude of the disturbance does not change. A magnified portion of the oscillation is shown in the inset to figure $1(b)$ from which it may be inferred that the disturbance is well-resolved. The same type of behaviour was observed when the number of streamwise mesh points was increased to 1601. Because the new form of the instability develops at an earlier time, it supercedes the more regular wave packet calculated by Cassel et al. (1995) which is then not observed on the finer spatial mesh.

Cassel et al. (1995) found that a wave packet first appeared on a coarser mesh at the corner for $\alpha \approx 3.9$. It has been determined here that, on a refined spatial mesh, small but visible persistent oscillations appear near the point of minimal wall shear for $\alpha$ as low as 3.7; note that this value and (and a subsequent critical value of $\alpha=4.2$ ) 

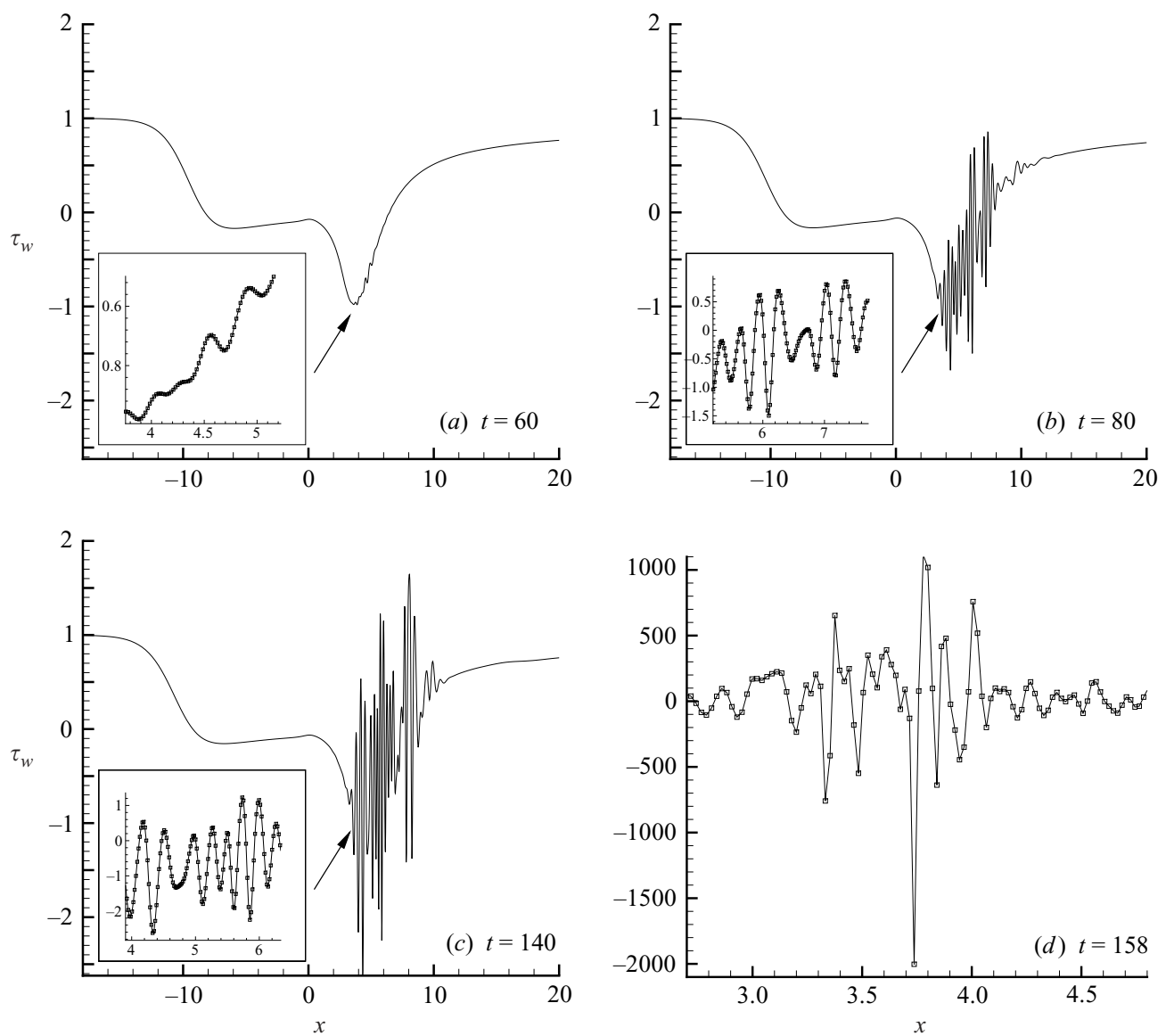

Figure 2. Temporal development of the instability for $\alpha=4.2$. The oscillations appear around $t=60$ but do not become unbounded until around $t=158$.

are approximate and might shift slightly depending on further grid refinement. For $\alpha<4.2$, the oscillations in the wall shear trough remain bounded for all time but for all $\alpha \gtrsim 4.2$, the instability was observed to grow spontaneously and without bound. Calculated results for the case $\alpha=4.2$ are shown in figure 2 where the insets show magnified pictures of the oscillations along with the grid points. At $t=60$ the onset of small oscillations may be seen downstream of the minimal shear point. The average amplitude of the disturbance grows rather slowly with time as the oscillation sloshes back and forth in the wall shear trough and by $t=140$, it is still $O(1)$. Shortly thereafter the disturbance enters a period of explosive growth and by $t=158$ the maximum amplitudes have grown through three orders of magnitude; note however that even at this stage the grid spacing is still reasonable. Since the instability that develops here does so at an essentially fixed streamwise location, it is an absolute instability.

It may be observed from figures 1 and 2 that the present disturbance exhibits oscillations of substantially higher frequency than the wave packet form of Cassel et al. (1995). The need for a sufficiently refined mesh has been pointed out by Tutty \& Cowley (1986) in order for some instabilities to appear and here the higher-order modes have only been revealed by using smaller streamwise mesh sizes. In the present 


$\begin{array}{rrcccc}I_{\max } & a & x_{0} & \Delta x_{x \sim-11} & \Delta x_{x=0} & \Delta x_{x \sim 5} \\ 301 & 10 & 0 & 0.23 & 0.1 & 0.13 \\ 801 & 5 & 0 & 0.12 & 0.02 & 0.04 \\ 1601 & 5 & 5 & 0.11 & 0.02 & 0.01\end{array}$

TABLE 1. Comparison of three different mesh spacings at key streamwise locations for $\alpha=4$ (the separation point, the ramp corner and near the downstream oscillations).

study, care was taken to ensure that the mesh sizes used near $x=0$ were at least as small as those employed by Cassel et al. (1995) and a comparison of some mesh sizes at certain streamwise locations that were used in the present and previous studies is presented in table 1.

\section{Convective instabilities}

In the course of the numerical work, each case considered was evaluated on a sequence of finer meshes in order to ascertain grid independence. In order to reduce the overall amount of computational time for those solutions that reached an apparently steady state, calculations were started on a fine mesh by interpolating the preceding coarser mesh solution. This was done using a standard cubic spline technique (see, for example, Press et al. 1992). This procedure reduced the computational times substantially and a steady state was typically reached on the finer mesh in around 20 time units, as opposed to the several hundred time units required when started from the flat-plate solution. However, one significant feature revealed by this process was that the wall shear distribution exhibited a significant perturbation in the form of a small spike just after the interpolation process and upstream of the ramp corner. This perturbation then was convected downstream, growing in size and reaching its largest amplitude as it passed through the point of minimum shear. At this stage the perturbation was observed to either become unbounded within the backflow zone or, upon reaching the reattachment zone, it was seen to decay while continuing to move in the downstream direction. This behaviour appears to be that associated with a convective instability.

In the work of Huerre \& Monkewitz (1985), it was found that an absolute instability can occur with an increase in a certain controlling parameter but that this event is generally preceded by convective instabilities. Professor P. Huerre (private communication) had suggested that an analogous situation might apply in the present case and consequently this issue was studied here. In the present problem, an absolute instability develops spontaneously for $\alpha \gtrsim 4.2$ and the issue is whether convective instabilities can occur for smaller values of $\alpha$. To address this point, a small pulse of the form

$$
\tau_{w}\left(\hat{x}_{i}\right)=\tau_{i 1}+\tilde{A} \exp \left(-\mu\left(\hat{x}_{i}-\hat{x}_{j}\right)^{2}\right),
$$

was introduced in the wall shear for solutions which had reached an apparently steady state. Here $\tilde{A}$ is the initial amplitude of the disturbance, $i$ denotes a point in the mesh with the disturbance initially centred on $\hat{x}_{j}$ and $\mu$ controls the initial spread; in most of the computations $\mu=10$ so that the introduced pulse was fairly compact.

When such a disturbance was introduced upstream of the separation point or downstream of the reattachment point, it was observed to convect downstream and decay rapidly with time. On the other hand, when the disturbance was introduced 

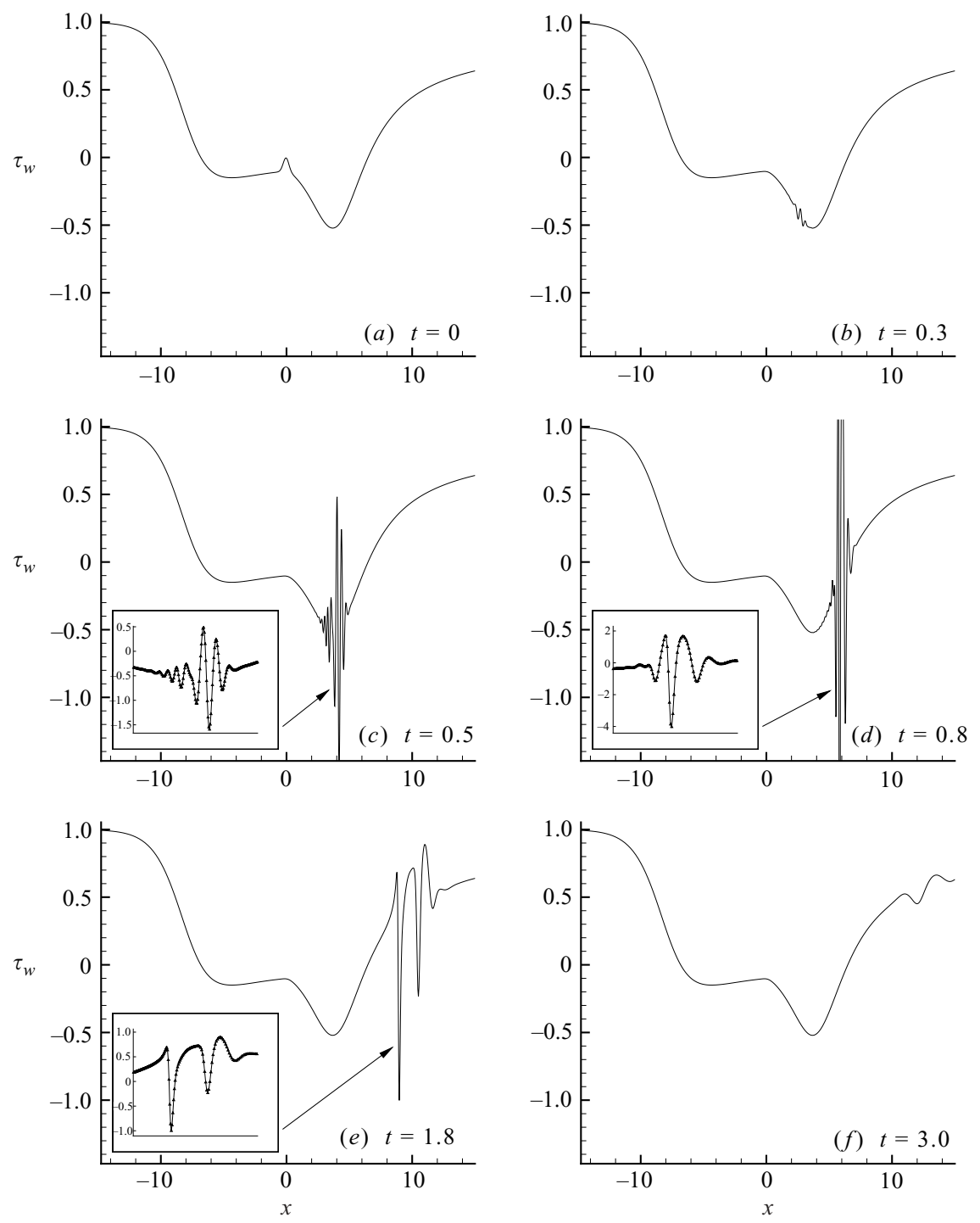

FIGURE 3. Temporal evolution of the wall shear for $\alpha=3.5$ for a disturbance introduced at $x=0$ with $A=0.1$ and $\mu=10$ showing the occurrence of convective instability. Note that the oscillation amplitude has been truncated in $(d)$.

at a station $\hat{x}_{j}$ within the reversed flow region, the behaviour was quite different (but at the same time reminiscent of that observed when a coarse mesh solution is interpolated to a smaller mesh). A typical development is shown in figure 3 for $\alpha=3.5$, where the initial pulse is shown in figure $3(a)$ with $\mu=10$ and $\tilde{A}=0.1$. After a short time, a wave packet is evident downstream of the corner in figure $3(b)$ which amplifies with time, particularly near the point of minimal shear in figure $3(c)$. The disturbance reaches maximum amplitude around $t \approx 0.80$ as shown in figure $3(d)$. As it is convected through the reattachment zone, the amplitude of the disturbance is substantially attenuated and subsequently decays as shown in figures $3(e)$ and $3(f)$. The corresponding development in the streamlines is shown in figure 4 . The 

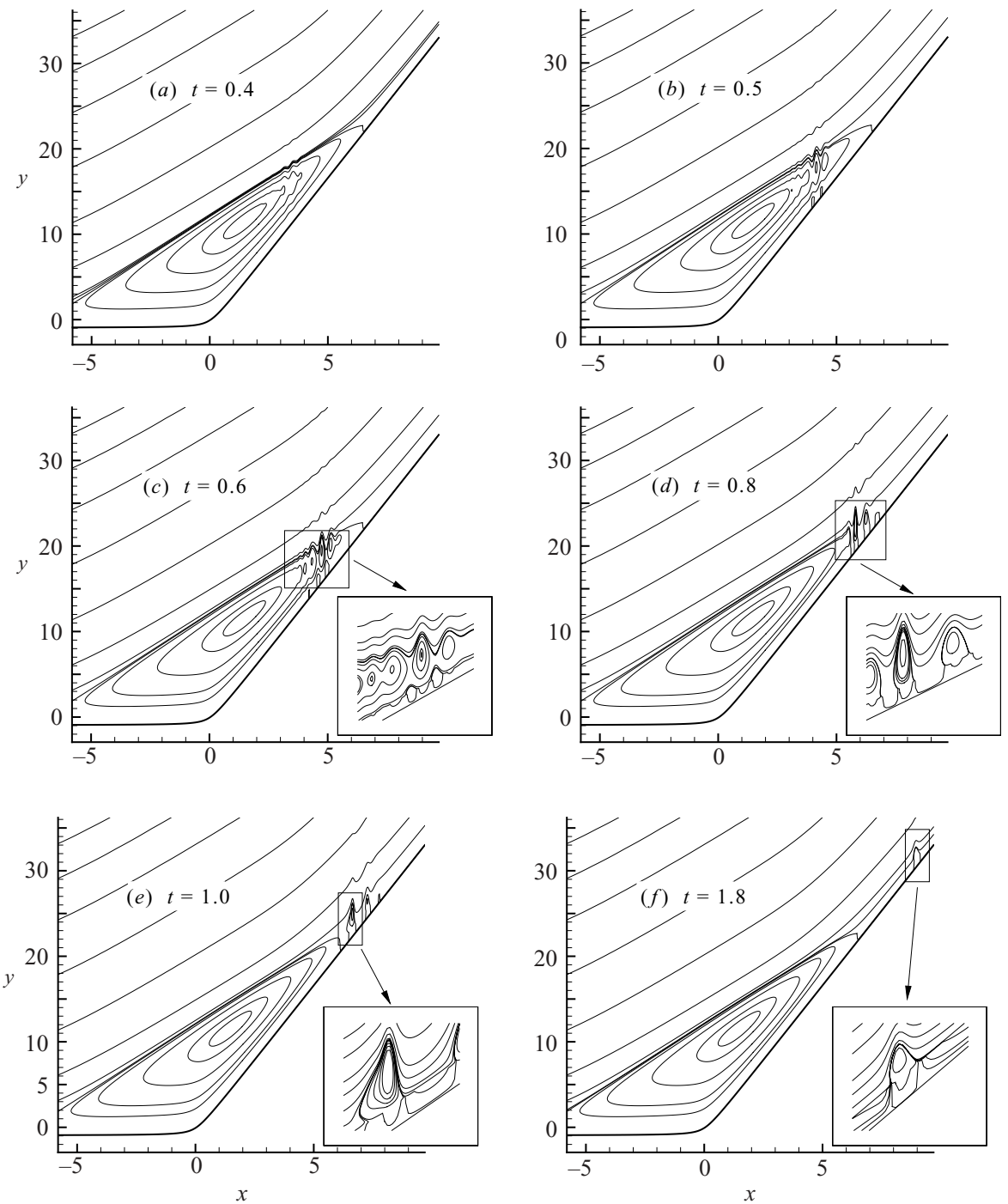

FIGURE 4. Temporal evolution of the instantaneous streamlines for $\alpha=3.5$ for a disturbance introduced at $x=0$ with $A=0.1$ and $\mu=10$.

development of small eddies near the surface before and near the reattachment zone is shown in detail in the insets. A similar phenomenon was seen in the triple-deck calculations of Tutty \& Cowley (1986) when flow past a vertically oscillating bump was computed. The reattachment zone here seems to act to 'chew up' the disturbance and as it convects downstream, rapid decay occurs.

The maximum amplitude of the oscillations at any downstream location, as well as the time required for the solution to return to the steady state, are influenced by the amplitude of the initial pulse. The maximum amplitude achieved at streamwise locations for $\alpha=3.5$ are shown in figure 5 for various values of $\tilde{A}$; here the vertical line indicates the streamwise location of the 'steady' reattachment point. Note that with increasing $\tilde{A}$, progressively larger amplitudes are reached upstream of reattachment. Further increases in $\tilde{A}$ lead to oscillations that become unbounded before the 


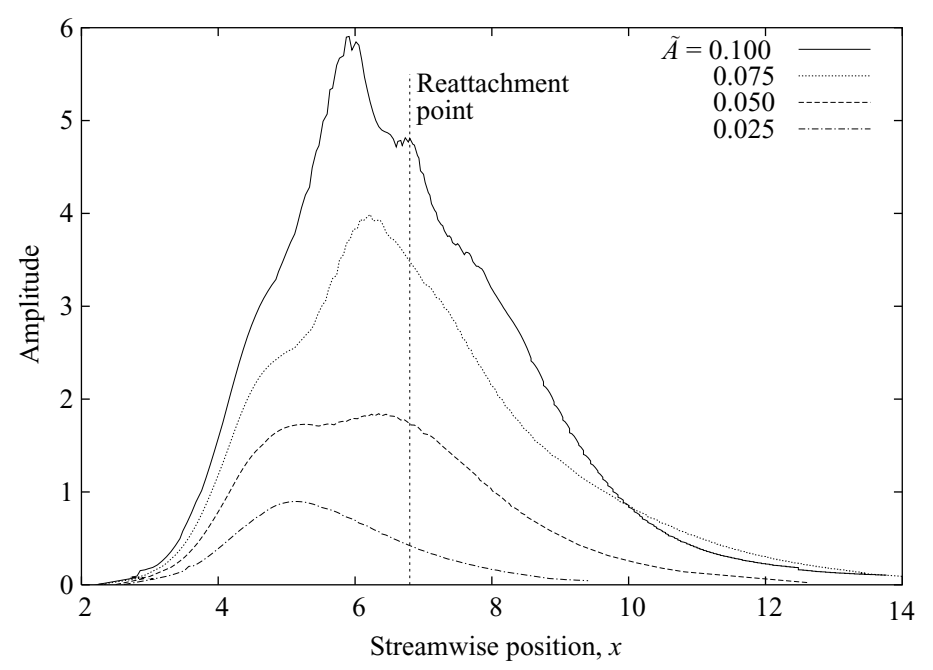

FIGURE 5. Maximum amplitude of computed oscillations in wall shear for a convective instability provoked by a pulse introduced at $x_{0}=0$ for $\alpha=3.5$ for various amplitudes $\tilde{A}$.

disturbance can be processed by the reattachment zone; for example, a disturbance with $\tilde{A}=0.5$ introduced at $x_{0}=0$ eventually achieves unbounded amplitude within the backflow zone. A precise value of $\tilde{A}$ for which unbounded oscillations occur cannot be determined uniquely because the threshold value depends on where the pulse is introduced; for example, a disturbance with $\tilde{A}=0.05$ introduced at $x_{0}=-4.8$ (just downstream of the separation point) also becomes unbounded. By contrast, perturbations introduced downstream of the point of minimum wall shear have a greatly reduced effect and, for example, a perturbation with $\tilde{A}=0.5$ introduced at $x_{0}=4$ (near the minimum shear point and in a zone of rising pressure) produced oscillations that were barely visible and which decayed rapidly. An important point is that in order for the disturbance to grow to unbounded amplitudes, it must be introduced upstream of the point of minimum shear so that it has sufficient time to develop and amplify.

In summary, convective instabilities were observed first at a critical value of $\alpha \approx 3.2$ and were provoked either through an interpolation process to a finer grid or via introduction of a disturbance like equation (5.1). As the ramp angle is increased from this value, the solutions become progressively more sensitive to perturbations of the form (5.1) in the reversed flow region; the observed phenomena have been described in some detail for $\alpha=3.5$. If the amplitude of the introduced perturbation at a given station is too large, the instability can reach unbounded amplitudes within the backflow zone. At a second critical value of $\alpha \approx 3.7$, the development of a spontaneous absolute instability was observed similar to that shown in figure 2 . However, in the range $3.7 \lesssim \alpha \lesssim 4.2$, the amplitude remains bounded and a type of quasi-steady state is reached. Finally for $\alpha$ greater than a third critical value of $\alpha \approx 4.2$, a spontaneous absolute instability occurs which ultimately reaches unbounded amplitudes.

\section{Theoretical considerations}

Rayleigh's inflection point theorem states that an inflection point in the streamwise velocity profile is a necessary condition for instability. Cassel et al. (1995) found inflection points near the corner for cases with $\alpha \geqslant 3.9$ and $\alpha=3.9$ was considered 


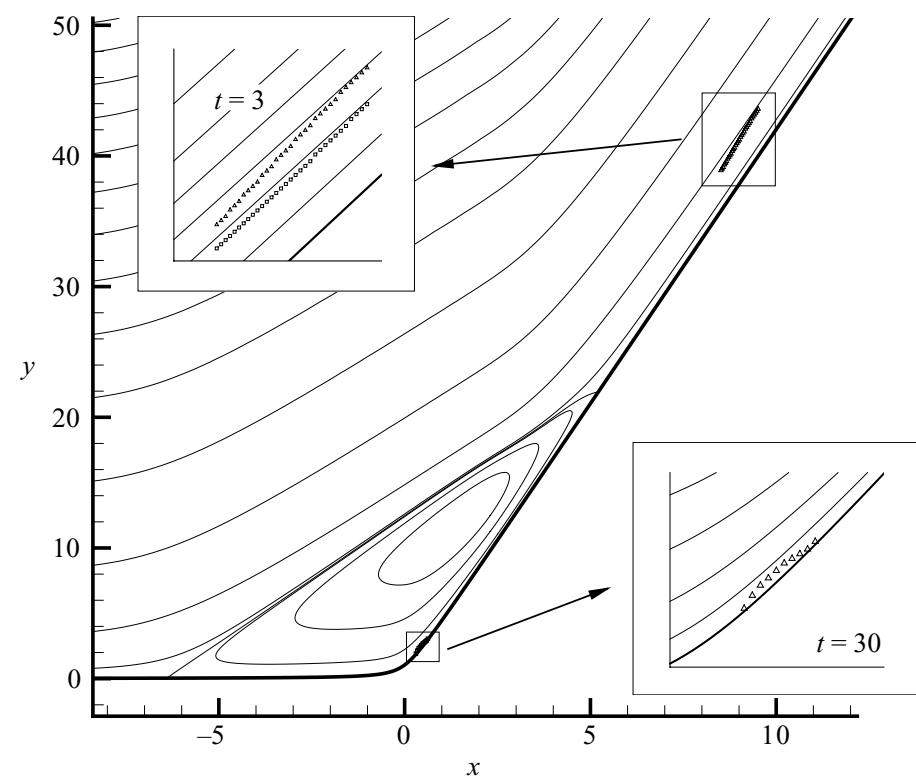

FiguRE 6. Instantaneous streamlines for $\alpha=4.2$ showing inflection points that occur near the corner at $t=30$ and far downstream at $t=3$.

to be the critical angle for the onset of instability. However, in the present study with much refined streamwise mesh spacings, small oscillations were observed in the wall shear for $\alpha=3.7$ and the flow appears to be convectively unstable for $\alpha$ as low as $\alpha=3.2$. The issue of the occurrence of inflection points in the range of numerical solutions was studied in detail.

Generally inflection points were found in two regions. At early times and well before $t_{d}$ (the time at which a monotonic decrease in $\partial \tau(x, 0) / \partial t$ was observed to end), inflection points were found far downstream of the corner and outside the recirculation zone, even for the cases at low ramp angle which ultimately reached a stable steady state. However, as the calculation continued these points passed downstream and out of the computational domain. Such inflection points are not believed to be important in relation to the issue of stability.

At larger ramp angles, inflection points were also found near the corner and by contrast these are believed to be significant. The first case where an absolute instability produces unbounded oscillations is for $\alpha=4.2$ and here inflection points appeared near the corner prior to $t_{d}$. In figure 6 the streamline patterns for $\alpha=4.2$ are shown at $t=30$, which is around the time inflection points appear near the corner. Note that the motion is unperturbed at this stage and oscillations do not appear subsequently until around $t=50$. The locations of downstream inflection points, that appeared around $t=3$ and subsequently are convected out of the domain, are also shown; by $t=30$, for example, these inflection points are no longer present.

Fjørtoft's theorem is a further necessary condition at any streamwise location for instability and is

$$
\frac{\partial^{2} u}{\partial y^{2}}\left\{u(y)-u\left(y_{i p}\right)\right\}<0,
$$

where $y_{i p}$ denotes the location of the inflection point and $y$ is any point on the profile. There are two possible cases, corresponding to the sign of $u\left(y_{i p}\right)$. The situation where 

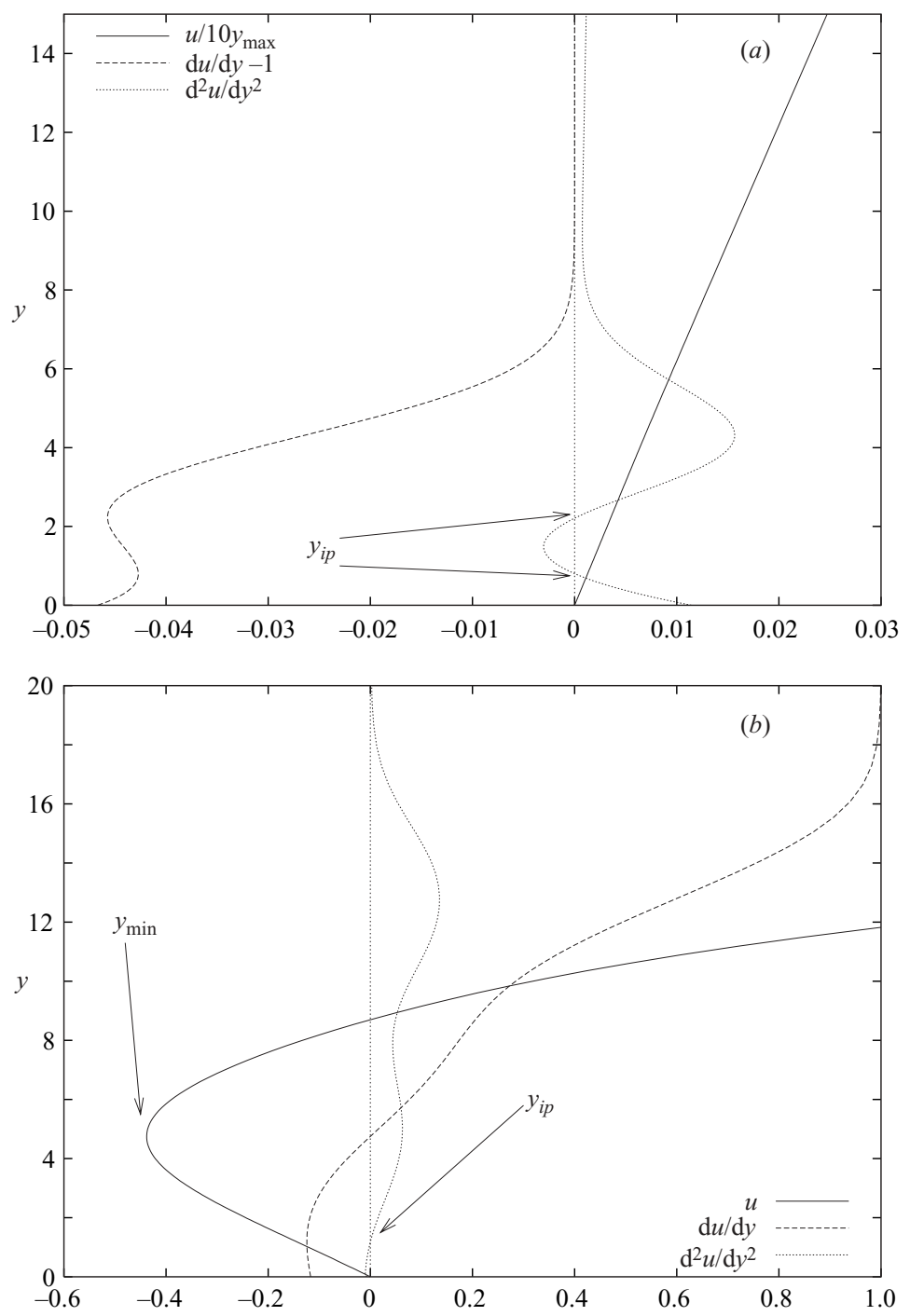

FIGURE 7. Velocity profiles and derivatives for $\alpha=4.2$ showing the locations of the inflection points. (a) A station downstream of the reversed flow zone at $t=3, x=9$.5. Here $u$ and $\mathrm{d} u / \mathrm{d} y$ have been scaled pragmatically to fit on one graph. (b) A station inside the reversed flow zone at $t=20, x=0.3$.

$u\left(y_{i p}\right)>0$ occurs for the inflection points that occur early in the calculations far downstream on the ramp. For these streamwise stations $\partial^{2} u / \partial y^{2}>0$ at the wall and in view of the no-slip condition at the wall, condition (6.1) is satisfied. Typical profiles are shown in figure 7(a). It may be observed that there are two inflection points in these downstream profiles. In figure 6, the locations of the inflection points where the curvature changes from positive to negative (in the increasing normal direction) are shown as squares; the inflection points where the curvature changes from negative to positive are shown as triangles. For $u\left(y_{i p}\right)<0$, which occurs in the reversed flow region, the inflection points occur near the wall and $\partial^{2} u / \partial y^{2}>0$ at the extremum point as shown in figure 7(b). Thus in both cases Fjørtoft's criterion is satisfied indicating that an instability is possible. 
The final and most important test for instability is to determine if equation (1.2) has solutions for the eigenvalue $c$ which have a positive imaginary part; as shown by Tutty \& Cowley (1986) this is a necessary and sufficient condition for instability. This test is much more difficult to apply to a time-dependent developing flow. The integral is difficult to evaluate accurately and here the algorithm of Tutty \& Cowley (1986) was used (as corrected by Cassel 1993) which employs Padé approximations over a portion of the integration range. Equation (B 2a) of Tutty \& Cowley (1986) is corrected here to read

$$
I_{1}-I_{0}=e h(1+d h)^{-1}+g d^{-1} \log (1+d h)+k h .
$$

Tutty \& Cowley (1986) obtained numerical solutions of the triple-deck equations using the interaction law $A=0$ for flow over a small bump on a surface and in these solutions were able to find a finite range in $x$ where $c_{i}>0$. On the other hand, in the computations of Cassel et al. (1995) for the compression ramp, it proved difficult to find any consistent pattern in the calculated results for $c_{i}$ despite many attempts. In fact the patterns that emerged in the present computations only became evident when the streamwise mesh was refined sufficiently.

The integral in equation (1.2) can be written in computational coordinates as

$$
J(x, t, c)=\int_{\hat{y}=0}^{\hat{y}=y_{\max }} b\left\{1-\frac{\Gamma(\hat{y})}{b} \frac{\partial u}{\partial \hat{y}}\right\} \frac{\mathrm{d} \hat{y}}{\Gamma(\hat{y})(u-c)^{2}}-\frac{1}{c}=0,
$$

where the numerical solution for $u$ is used in this equation at any $x$ and $t$. Tutty \& Cowley (1986) used a secant method to find possible eigenvalues and this method was also attempted here. At any streamwise station for fixed $t, J$ was first evaluated for two different values of $c$ and these are used to initiate the search for the zero. The success of this method is highly dependent on the choice of initial guesses. The method was found to be prone to diverge, even when a zero located at an upstream station was used to initiate a search at the current streamwise station. In addition there may be more than one eigenvalue at each station and the additional roots can only be found by taking various initial guesses in the complex $c$-plane. This process proved to be quite time consuming and frustrating because of the tendency of the scheme to diverge. Thus an alternative process was adopted to find the roots wherein the surface $|J|$ was studied instead. This function is positive and the roots are defined where $|J|$ just touches the complex $c$-plane. Good initial guesses for the roots are easily obtained by looking at a contour plot of $|J|$. The numerical procedure used to find the roots is the Downhill Simplex Method (see Press et al. 1992). The method is fairly slow and requires many more function evaluations than the secant method but it is also quite robust. The scheme is initiated with a triangle of complex numbers $c$ (i.e. a simplex) and at each step in the process the triangle is deformed to move closer to the root. Normally the point in the triangle at the highest altitude on the surface $|J|$ is reflected through the line joining the other two; a number of other measures are also taken so that the algorithm can successfully negotiate flat or narrow valleys in the surface. The termination criterion of the search was based on the predicted size of the next step such that convergence was deemed to have occurred when this value was less than $10^{-15}$. Note however that this search process merely locates a local minimum in $|J|$ and does not guarantee that a zero has been located. In practice, an eigenvalue was considered to have been found when $|J|<10^{-4}$ at an extremum. The roots usually occur at sharp holes in the surface and the function value there was usually zero to machine precision. Thus there was no ambiguity as to whether a 
root had actually been found. As a further check the secant method used by Tutty \& Cowley (1986) was also used with initial guesses taken near the results obtained with the present method; under these circumstances the secant method converged rapidly to the same answer.

For low ramp angles such as $\alpha=1,2$ a stable steady state was reached in the computations and the steady profiles were examined to determine if there were any locations where $c_{i}>0$. An extensive search of the results obtained on $(801,151)$ and $(1601,151)$ meshes did not find any such roots. For the cases that were observed to become unstable computationally, roots of equation (6.2) were found and it is useful to first examine the case $\alpha=5$ at times before the absolute instability is well-developed (for this case visible small spontaneous oscillations appear at $t \approx 22$ and become large at $t \approx 32$ before becoming unbounded at $t \approx 76$ ). In figure $8, c_{i}$ for the obtained roots is plotted versus the physical streamwise coordinate at $t=20$ and $t=26$; the wall shear is also plotted for reference. At $t=20$, the wall shear is smooth except for a small distortion that has developed just upstream of $x=2$ and, as shown in figure $8(a)$, roots indicating instability have been found near the ramp corner. The solid lines in this graph denote actual roots such that $|J|<10^{-4}$ and are labelled $1,2 a$ and $2 b$. The dotted lines to either side of these curves are points where the simplex method has converged but only to a local minimum of $|J|$; note that the solid and dotted lines appear to be continuous at their juncture. At a subsequent time $t=26$, shown in figure $8(b)$, the wall shear has developed a smooth oscillation downstream of the point of minimum shear and a new curve labelled 3 has appeared. Note that curves $1,2 a$ and $2 b$ are still present but have moved upward somewhat, suggesting that somewhat larger growth rates can occur at this later time. The growth rates associated with curve 3 are lower than for the other curves but it is believed that this curve is the precursor of an absolute instability, whereas the others are indicative of convective instabilities. Curve 3 does not quite reach the axis since the integral (5.1) is improper when $c$ is real. The curves shown in figure 8 are all located near the ramp corner whereas the development of growing oscillations eventually occurs near and downstream of the point of minimum shear. Roots were found near the point of minimum shear and these are also shown in figure $8(b)$ as single points; in general no consistent pattern was observed for the roots in this zone, although the pattern suggests the presence of multi-frequency oscillations as observed in the computations. In figure 9 contour plots of the surface $|J(c)|$ are shown at the three streamwise locations indicated in figure $8(b)$ by broken vertical lines. These plots show the holes (depicted as dark) where a root occurs. The labelling of the roots corresponds to that in figure $8(b)$.

In this study the occurrence of a spontaneous absolute instability was first observed for $\alpha=3.7$ and it is of interest to try to correlate the patterns seen in figure 8 and 9 with those observed in the computations. For $\alpha \leqslant 2$ there are no roots of equation (6.2). For $\alpha=3$ some roots were observed but they are sparsely scattered and do not form continuous curves as in figure $8(a)$; in addition the growth rates are small with $c_{i}<0.1$. For $\alpha=3.2$, partial forms of curves 1 and $2 a, b$ are present and by $\alpha=3.4$ these curves are complete. The computational results suggested that $\alpha=3.0$ is stable; on the other hand for $\alpha=3.2$ the flow exhibits a weak convective instability that grows in strength as $\alpha$ increases to 3.4. Thus it appears that curves 1 and $2 a, b$ are associated with the observed convective instability.

For $\alpha=3.6$, a portion of curve 3 starts to appear in the vicinity of the eventual maximum and by $\alpha=3.7$ (at a time just prior to the onset of absolute instability and when small oscillations are present) the evolution is essentially complete and 

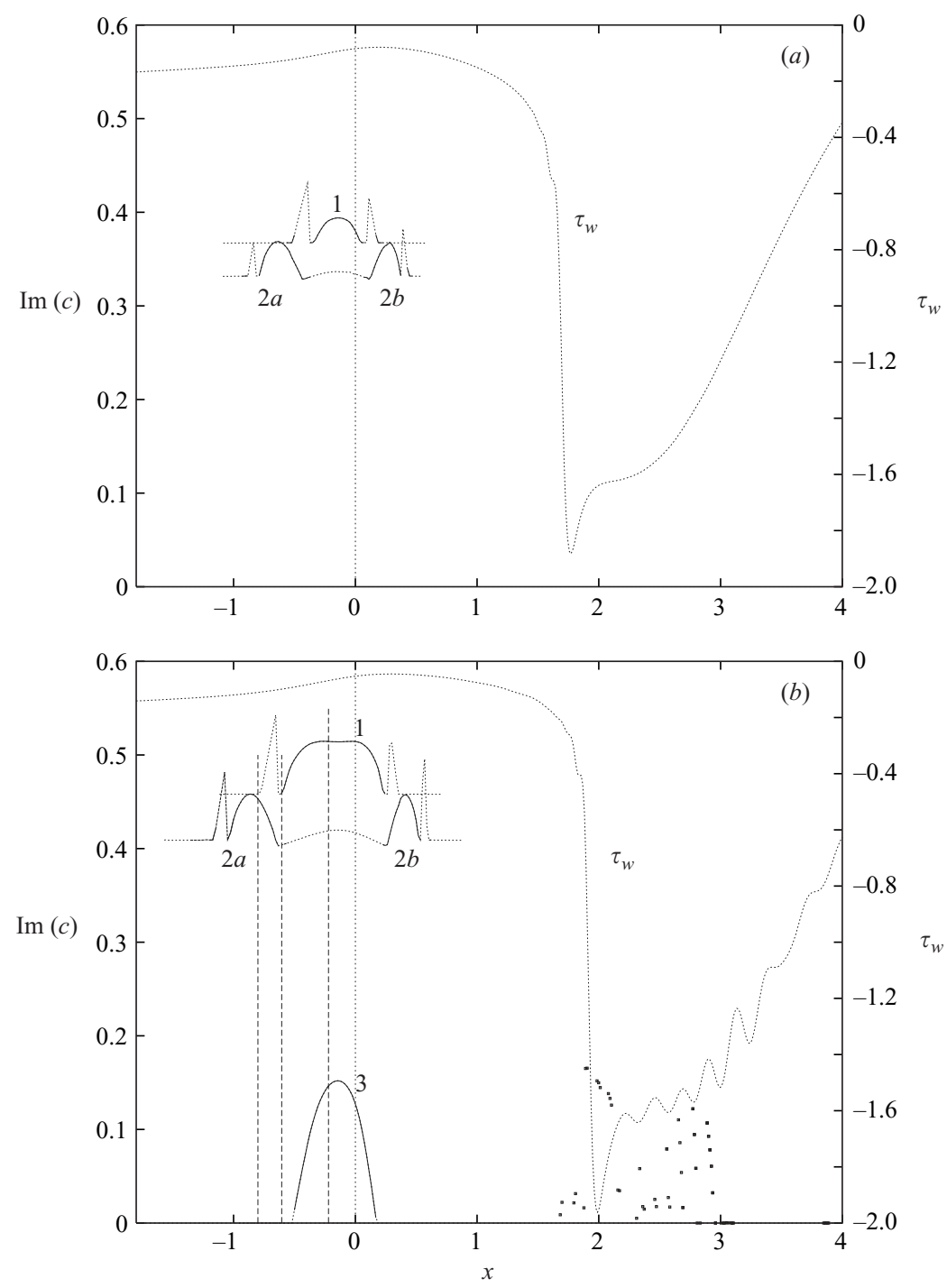

FIGURE 8. Imaginary part of the roots of $J(c)=0$ together with the wall shear for $\alpha=5$. (a) $t=20,(b) t=26$.

qualitatively similar to that shown in figure $8(b)$, with curve 3 extending almost to the axis $c_{i}=0$. For these reasons, it is believed that the evolution of curve 3 is associated with the onset of absolute instability. The case $\alpha=3.6$ reaches an apparently steady state but the 'steady state' is characterized by small oscillations in the wall shear trough that require a long time to develop; this case is believed to be on the verge of producing absolute instability. It may be noted that the growth rates associated with curves 1 and $2 a, b$ are larger than those associated with curve 3 and this is consistent with computational experience. The convective instabilities were observed to grow fairly rapidly whereas a spontaneous absolute instability grows initially slowly and then plateaus for a time before becoming unbounded.

It is of interest to attempt to compare the actual growth of the disturbances with the predicted growth rates of the form $\delta \exp \left(k c_{i} t\right)$, where $k=2 \pi / \lambda$ is the wavenumber 

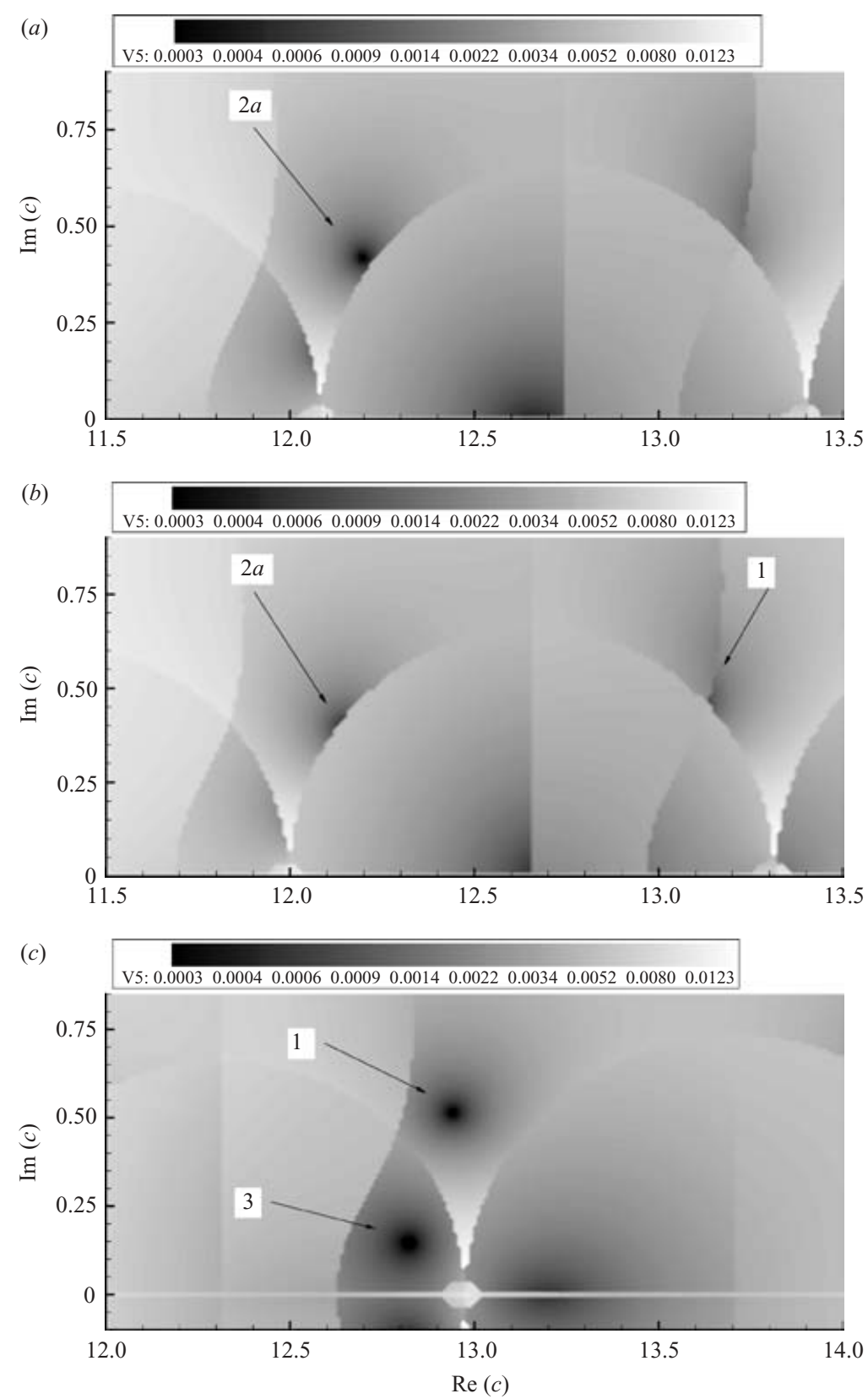

Figure 9. The surface $|J(c)|$ for $\alpha=5$ at $t=26$. (a) $x=-0.80,(b) x=-0.60,(c) x=-0.22$.

and $\lambda$ is the wavelength with $c_{i}$ estimated from figure 8(a). For $\alpha=5$, oscillations were first visible in the wall shear near the minimum for $t \approx 22$ and an estimate of $\lambda=0.286$ was made at $x=4$; an estimate of the maximum value of $c_{i}=0.12$ was obtained at this location and time from a graph similar to figure $8(a)$. These considerations predict a growth of the disturbance of the form $\delta \exp (2.74 t)$. The actual growth of the disturbance is shown in figure 10, where the maximum amplitude is plotted versus time. A curve fit to the early results produced $2.7 \times 10^{-10} \exp (0.744 t)$. This, of 


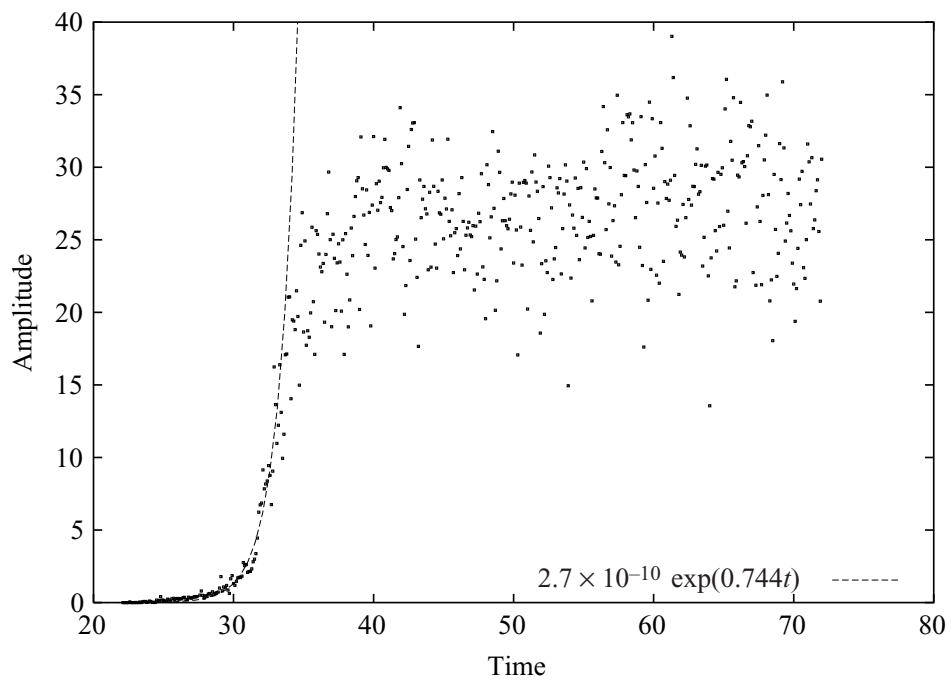

FIGURE 10. Maximum amplitude of the disturbance versus time for $\alpha=5$, together with a least-squares curve fit to early growth data.

course, is not close agreement with the predicted growth rates but it is at least of comparable magnitude. Refining the comparison is difficult because the wavelengths of the disturbance were observed to decrease as the disturbance grew. The subsequent development of the disturbance in figure 10 shows a complicated plateau behaviour as it sloshes back and forth in the wall shear trough; eventually unbounded oscillations occur around $t=76$.

\section{Conclusions}

The evolution of an absolute instability in supersonic boundary-layer flow over a compression ramp has been documented and connected with the computed eigenvalues of the relation given by Tutty \& Cowley (1986), that is a necessary and sufficient condition for instability. The evolution of this instability and a consistent pattern in the calculated eigenvalues was evident only when the streamwise mesh was sufficiently refined. As the ramp angle is increased from zero through to a critical value of around 3.2, the lack of eigenvalues and the computed solutions, including those with reversed flow, show that the two-dimensional boundary layer flow is steady and stable. There is then a parameter range $(3.2 \lesssim \alpha \lesssim 3.7)$ where the flow apparently reaches a stable steady state but, in fact, the motion is convectively unstable in the reversed flow region near the ramp corner. If a disturbance of sufficiently large amplitude is introduced in the backflow zone, an absolute instability can occur in this range. As the ramp angle is increased to higher values, at around a scaled value of $\alpha \simeq 3.7$, a spontaneous absolute instability occurs with bounded amplitude; for $\alpha \gtrsim 4.2$ the amplitude eventually becomes unbounded. The present results are in agreement with the work of Huerre \& Monkewitz (1985) which suggests that as the controlling parameter is increased a region of convective instability precedes that where absolute instability occurs.

The authors would like to acknowledge helpful discussions with Dr S. J. Cowley and Professor P. Huerre. 
This study was initiated by David Walker and, despite his illness, the project was driven forward by David's enthusiasm and energy.

\section{REFERENCES}

Ackeret, J., Feldmann, F. \& Rott, N. 1947 Investigations of compression shocks and boundary layers in gases moving at high speed. NACA Tech. Memo. 1113. ETH Zurich No. 10.

Burggraf, O. R., Rizzetta, D., Werle, M. J. \& Vatsa, V. N. 1979 Effect of Reynolds number on laminar separation of a supersonic stream. AIAA J. 17, 336-343.

CASSEL, K. W. 1993 The effect of interaction on boundary-layer separation and breakdown. PhD thesis, Lehigh University.

Cassel, K. W., Ruban, A. I. \& Walker, J. D. A. 1995 An instability in supersonic boundary-layer flow over a compression ramp. J. Fluid Mech. 300, 265-286.

Chapman, D. R., KueHn, D. M. \& Larson, H. K. 1957 Investigation of separated flows in supersonic and subsonic streams with empasis on the effect of transition. Tech. Note. 3869. NACA.

Degani, A. T., Li, Q. \& Walker, J. D. A. 1996 Unsteady separation from the leading edge of a thin airfoil. Phys. Fluids 8, 704-714.

Huerre, P. \& Monkewitz, P. A. 1985 Absolute and convective instabilities in free shear layers. J. Fluid Mech. 159, 151-168.

Huerre, P. \& Monkewitz, P. A. 1990 Local and global instabilites in spatially developing flows. Annu. Rev. Fluid Mech. 22, 473-537.

Korolev, G. L., GajJar, J. S. B. \& Ruban, A. I. 2002 Once again on the supersonic flow separation near a corner. J. Fluid Mech. 463, 173-199.

LiepmanN, H. W. 1946 The interaction between boundary layers and shock waves in transonic flow. J. Aero. Sci. 13, 623-637.

Messiter, A. F. 1983 Boundary-layer interaction theory. Trans. ASME E: J. Appl. Mech. 50, 11041113.

NeILAND, V. Y. 1969 On the theory of laminar boundary-layer separation in supersonic flow. Izv. Akad. Nauk SSSR, Mekh. Zhid. i Gaza 4, 53-57.

NeILAND, V. Y. 1970 Upstream propogation of perturbations in a hypersonic flow interacting with a boundary layer. Izv. Akad. Nauk SSSR, Mekh. Zhid. i Gaza 4, 40-49.

NeILAND, V. Y. 1981 On the theory of laminar boundary-layer separation in supersonic flow. Adv. Mech 4, 3-62.

Press, W. H., Teukolsky, S. A., Vetterling, W. \& Flannery, B. P. 1992 Numerical Recipes in Fortran. Cambridge University Press.

Rizzetta, D. P., Burggraf, O. R. \& Jenson, R. 1978 Triple-deck solutions for viscous supersonic and hypersonic flow past corners. J. Fluid Mech. 89, 535-552.

Ruban, A. I. 1978 Numerical solution of the local asymptotic problem of the unsteady separation of a laminar boundary layer in a supersonic flow. USSR Comput. Maths. Math. Phys. 18, $175-187$.

Smith, F. T. \& Khorrami, A. 1991 The interactive breakdown in supersonic ramp flow. J. Fluid Mech. 224, 197-215.

Stewartson, K. 1974 Multistructured boundary layers on plates and related bodies. Adv. Appl. Mech. 14, 145-239.

Stewartson, K. \& Williams, P. G. 1969 Self-induced separation. Proc. R. Soc. Lond. A 312, 814-819.

Sychev, V., Ruban, A. I., Sychev, V. \& Korolev, G. 1998 Asymptotic Theory of Separated Flows. Cambridge University Press.

TutTy, O. R. \& Cowley, S. J. 1986 On the stability and the numerical solution of the unsteady interactive boundary-layer separation. J. Fluid Mech. 168, 431-456. 